\title{
Searching for water ice in the coma of interstellar object 2I/Borisov ${ }^{\star}$
}

\author{
Bin Yang (杨涁) $)^{1}$, Michael S. P. Kelley ${ }^{2}$, Karen J. Meech ${ }^{3}$, Jacqueline V. Keane ${ }^{3}$, Silvia Protopapa ${ }^{4}$, and \\ Schelte J. Bus ${ }^{3}$
}

1 European Southern Observatory, Alonso de Cordova 3107, Vitacura, Santiago, Chile e-mail: byang@eso.org

2 Department of Astronomy, University of Maryland, College Park, MD 20742-2421, USA

3 Institute for Astronomy, 2680 Woodlawn Drive, Honolulu, HI 96822, USA

${ }^{4}$ Southwest Research Institute, Boulder, CO 80302, USA

Received 17 November 2019 / Accepted 10 December 2019

\begin{abstract}
Aims. Interstellar objects passing through our Solar System offer a rare opportunity to probe the physical and chemical processes involved in solid body and planet formation in extrasolar systems. The main objective of our study is to search for diagnostic absorption features of water ice in the near-infrared (NIR) spectrum of the second interstellar object 2I/2019 Q4 (Borisov) and compare its ice features to those of the Solar System icy objects.

Methods. We observed 2I in the NIR on three separate occasions. The first observation was made on 2019 September 19 UT using the SpeX spectrograph at the $3 \mathrm{~m}$ IRTF and again on September 24 UT with the GNIRS spectrograph at the 8m GEMINI telescope; the last observation was made on October 09 UT with IRTF.

Results. The spectra obtained from all three nights appear featureless. No absorption features associated with water ice are detected. Spectral modeling suggests that water grains, if present, comprise no more than $10 \%$ of the coma cross section. The comet consistently exhibits a red D-type like spectrum with a spectral slope of about $6 \%$ per $1000 \AA$, which is similar to that of $1 \mathrm{I} /{ }^{\prime}$ Oumuamua and is comparable to Solar System comets.
\end{abstract}

Key words. techniques: spectroscopic - comets: individual: 2I/Borisov

\section{Introduction}

Understanding how planetary systems form from protoplanetary disks is one of the hottest topics in astronomy today. Solar system formation models show that as giant planets form, large numbers of planetesimals are ejected into the interstellar medium (Walsh et al. 2011; Shannon et al. 2015; Raymond et al. 2018). Consequently, a large number of extrasolar planetesimals must be traversing through interstellar space, and some eventually pass through our Solar System. These interstellar objects (ISOs) are expected to be icy (i.e., comet-like) with only a small fraction being rocky objects (Meech et al. 2016; Engelhardt et al. 2017). The mass distribution and chemical abundances within our Solar System are well studied; instead, the knowledge of other planetary systems is highly limited. Whether the Solar System is typical of planetary systems in general is an enduring question. ISOs provide a rare opportunity to study the details of planet-building processes in extrasolar planetary systems by delivering material to our Solar System for in-depth observations.

The first known ISO, 1I/'Oumuamua, was discovered on 2017 October 19, and an extensive 2.5-month follow-up campaign was conducted (Meech et al. 2017). Astrometric measurements from the ground and HST showed a $30 \sigma$ detection

\footnotetext{
* The spectra are also available at the CDS via anonymous ftp to cdsarc.u-strasbg.fr (130.79.128.5) or via http://cdsarc. u-strasbg.fr/viz-bin/cat/J/A+A/634/L6
}

of a radial nongravitational acceleration varying as $\sim r^{-2}$. Several hypotheses to explain this acceleration were tested and ruled out. The only feasible explanation for the acceleration requires a modest level of cometary outgassing (Micheli et al. 2018). Therefore, $1 \mathrm{I}$ is likely an icy body in spite of its asteroidal appearance. However, spectroscopic observations from 0.3 to $2.5 \mu \mathrm{m}$ did not reveal any diagnostic absorption features of water ice or hydrated minerals (Fitzsimmons et al. 2018). The measured spectral slopes of $1 \mathrm{I}$ in the optical wavelength region vary from $10 \% / 100 \mathrm{~nm}$ to $17 \% / 100 \mathrm{~nm}$ (Ye et al. 2017; Fitzsimmons et al. 2018), which are interpreted as low-albedo organic compounds that have undergone exposure to cosmic rays (Fitzsimmons et al. 2018).

C/2019 Q4 (Borisov) was discovered on 2019 August 30 and quickly found to be on a hyperbolic orbit (MPEC R106; Sep. 11, 2019) suggesting an interstellar origin. A dynamical analysis indicates that this eccentricity was not caused by an interaction with a giant planet, since the high eccentricity remains when the orbit is integrated backward to a time before its entrance into the Solar System. Observational effects, or cometary outgassing, are also insufficient to explain the extreme orbital eccentricity of the object, making C/2019 Q4 the second known ISO, designated as 2I/Borisov (MPEC S71; 2019 Sep. 24). Unlike 1I/'Oumuamua, 2I displays a dust coma at 2.8 au from the Sun, typical of Solar System comets at this distance. Fitzsimmons et al. (2019) reported the first detection of the $\mathrm{CN}(0-0)$ emission band at $3880 \AA$ in the optical spectrum of 2I. Opitom et al. (2019) 
confirmed the $\mathrm{CN}$ detection, but concluded that 2I is highly depleted in $\mathrm{C}_{2}$. Current observations of 2I suggest that it contains volatiles and that its composition may be similar to Solar System carbon-chain depleted comets.

Water ice, one of the planetary-building blocks, is of great importance to the formation of planets (A'Hearn 2011). The presence of water ice enhances solid surface density and increases sticking efficiency, which in turn, catalyzes the rapid formation of planetesimals and decreases the timescale of giant planet core accretion (Min et al. 2016). Ices are sensitive to changes induced by thermal and radiation processing, so their characteristics and abundances can provide central clues to those aspects of planetary heritage (Mumma \& Charnley 2011). The solid state features of water ice are influenced by its formation temperature and thermal history (Grundy \& Schmitt 1998, and references therein). Water ice is commonly detected via the distinctive infrared absorption bands at 1.5 and $2.0 \mu \mathrm{m}$. Amorphous and crystalline water ice are easily distinguishable at temperatures $\lesssim 170 \mathrm{~K}$; crystalline ice exhibits a narrow feature at $1.65 \mu \mathrm{m}$, which is absent in amorphous ice. The feature is suppressed as temperature increases (Grundy \& Schmitt 1998). Studying the relative strength and shape of the water ice features in the coma can yield important information on the impurity, physical structure, and the temperature of ice grains. In turn, we may be able to set constraints on the processes experienced by 2I and its formation conditions.

There are three objectives of our study: (1) to search for diagnostic absorption features of water ice and other compounds (such as hydrated minerals or organic materials) in the coma or on its surface; (2) to compare water ice features in 2I to other Solar System icy objects (e.g., comets, Centaurs, and Kuiper Belt objects) and set constraints on its birth conditions; (3) to search for the ultra-red organic materials that may be present on the surface of 2I or in the coma as a result of long-term exposure to Galactic cosmic rays or UV bombardment.

\section{Observations and data reduction}

We observed 2I on three separate occasions. The first two observations were made using director discretionary time allocations. The first observation was carried out in the morning twilight on 2019 September 19 UT using the SpeX spectrograph at the $3 \mathrm{~m}$ IRTF telescope. The second observation was made on September 24 UT with the Gemini Near-InfraRed Spectrograph (GNIRS) spectrograph on the $8 \mathrm{~m}$ GEMINI telescope. The last observation was made in service mode, again with the SpeX/IRTF, on October 9. The observing geometry is shown in Table 1.

The SpeX observations were made with the high-throughput prism mode $(0.8-2.5 \mu \mathrm{m})$ and a $0.8^{\prime \prime} \times 15^{\prime \prime}$ slit that provides a spectral resolving power of $R \sim 150$ (Rayner et al. 2003). The GNIRS observations were taken with the short camera (plate scale: $0.15^{\prime \prime} \mathrm{pix}^{-1}$ ). The SXD cross-dispersed mode, $321 \mathrm{~mm}^{-1}$ grating and $1.0^{\prime \prime}$ slit, provides an averaged resolving power of $R \sim 1700$ over $0.9-2.5 \mu \mathrm{m}$ (Elias et al. 2006). At least one nearby G-type star was observed together with the comet during each run, which was used both as the telluric correction standard star and the solar analog. The SpeX data were reduced using the reduction pipeline SpeXtool (Cushing et al. 2004). The GNIRS data were reduced using the Image Reduction and Analysis Facility (IRAF) software and the Gemini IRAF package. Taking advantage of the similarity between the SpeX data and GNIRS data, we used the SpeXtool xtellcor routine for telluric correction and the xmergeorders routine for merging the GNIRS SXD spectra.
Table 1. Journal of the 2I observations.

\begin{tabular}{llccccc}
\hline \hline $\begin{array}{l}\text { UT Date } \\
\text { 2019 }\end{array}$ & Facility & $\begin{array}{c}r_{h}^{\dagger} \\
{[\mathrm{au}]}\end{array}$ & $\begin{array}{c}\Delta^{\dagger} \\
{[\mathrm{au}]}\end{array}$ & $\begin{array}{c}\alpha^{\ddagger} \\
{[\mathrm{deg}]}\end{array}$ & $\chi^{\S}$ & Standard \\
\hline Sep 19 & IRTF & 2.67 & 3.26 & 15.78 & 1.95 & HD 283886 \\
Sep 24 & Gemini & 2.60 & 3.16 & 16.77 & 2.15 & SAO 79667 \\
Oct 09 & IRTF & 2.41 & 2.84 & 19.78 & 1.77 & HD 87680 \\
\hline
\end{tabular}

Notes. ${ }^{(\dagger)} r_{h}$ and $\Delta$ are the heliocentric and geocentric distances, respectively; ${ }^{(\ddagger)} \alpha$ is the phase angle; ${ }^{(\S)} \chi$ is the airmass.

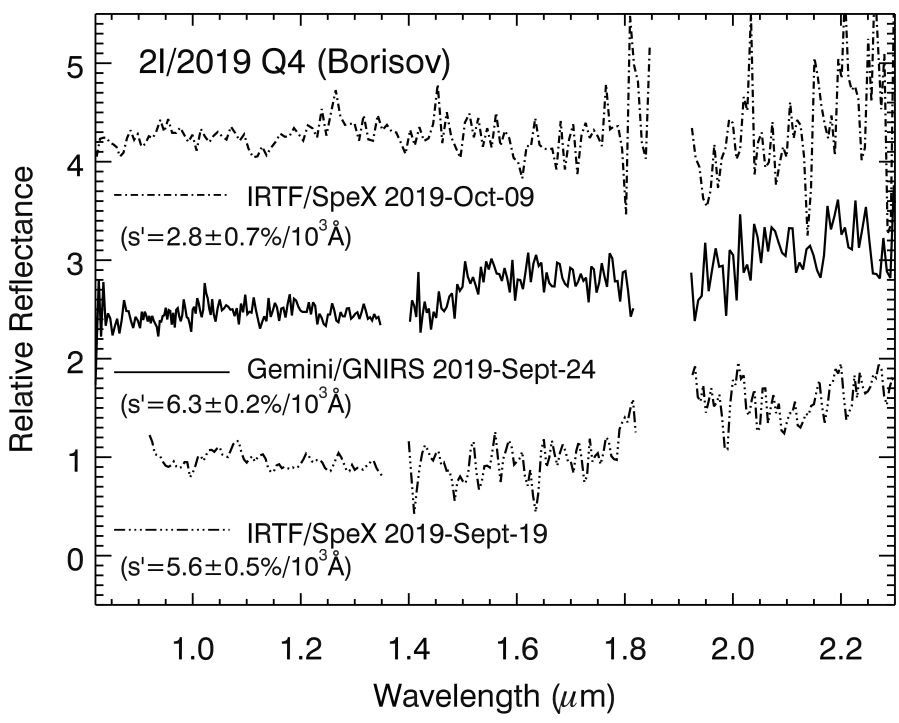

Fig. 1. Near-IR spectra of 2I/Borisov, taken with the Gemini-N and IRTF telescopes. The wavelength regions that are severely affected by the atmospheric absorption are omitted.

\section{Results}

The NIR observations of 2I are presented in Fig. 1. Although the three spectra were taken on different dates and with different instruments, they consistently appear featureless and show moderately red spectral slopes. We did not detect the diagnostic absorption bands of water ice at 1.5 and $2.0 \mu \mathrm{m}$. Among the three NIR observations, the Gemini dataset has the highest signal-tonoise ratio $(\mathrm{S} / \mathrm{N})$. Thus, we use the Gemini spectra for further analysis.

\subsection{Upper limits on the water ice abundance}

Previous water ice detections in the cometary comae show that icy grains consist of submicron- to micron-sized fine particles (Protopapa et al. 2014; Yang et al. 2014). However, optical observations suggest that the coma of $2 \mathrm{I}$ is dominated by large particles. For example, Jewitt \& Luu (2019) and Ye et al. (2019) consistently suggest that the effective size of the dust particles is about $100 \mu \mathrm{m}$. To estimate an upper limit on the amount of possible ice grains in the coma of $2 \mathrm{I}$, we synthesized a pure water ice spectrum using an effective particle radius of $1 \mu \mathrm{m}$, $10 \mu \mathrm{m}$, and $100 \mu \mathrm{m}$, respectively, based on the Hapke model (Hapke 2012). We adopted the optical constants of crystalline ice at $150 \mathrm{~K}$ from Mastrapa et al. (2008). We then created an ice mixture using amorphous carbon as a proxy for coma dust. The carbon spectrum is generated in the same way as the ice spectrum, using an effective particle radius of $100 \mu \mathrm{m}$ and the optical constants from Rouleau \& Martin (1991). Focusing on the water 


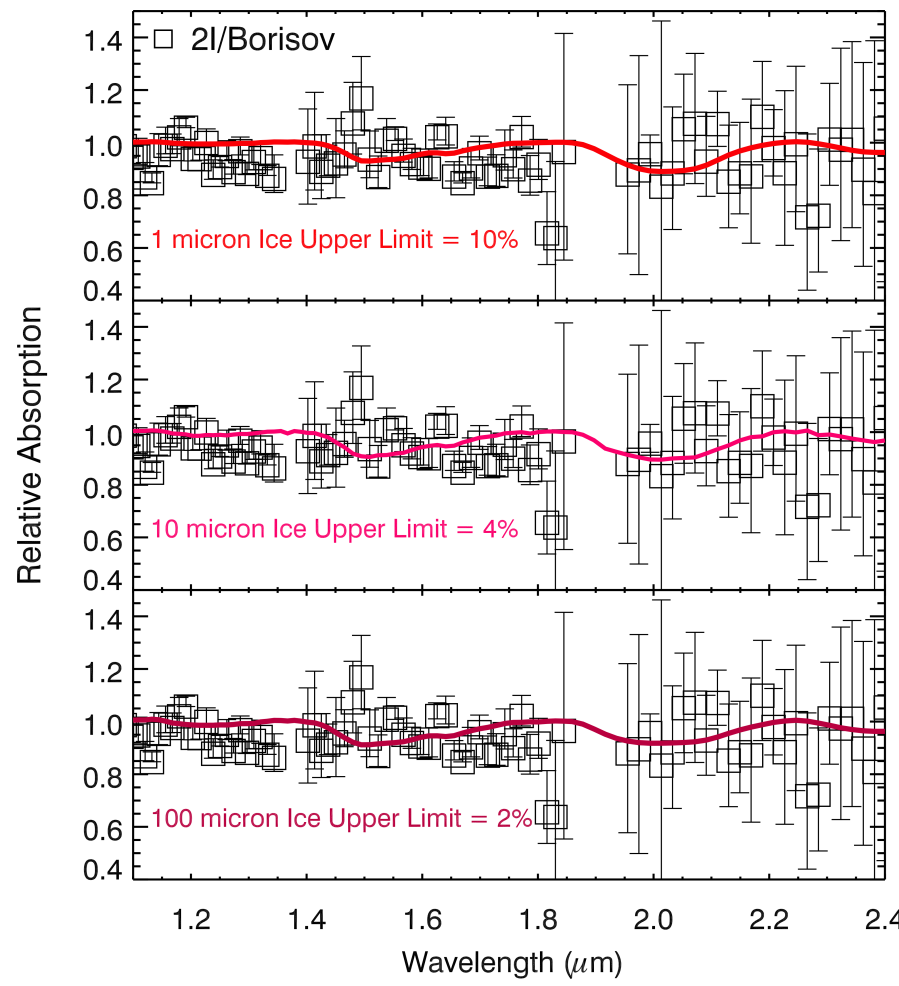

Fig. 2. Gemini spectrum of 2I/Borisov obtained on 2019 September 24 UT. The spectrum was re-sampled to a resolution of $\sim 60$ (open squares) and binned by a factor of 30 , and the continuum of the 2I spectrum was removed using a low-order polynomial model. The red lines are the modeled water ice mixture spectrum using an average particle size of $1 \mu \mathrm{m}, 10 \mu \mathrm{m}$, and $100 \mu \mathrm{m}$ (from top to bottom). Our model suggests that the water ice present in the coma is no more than $10 \%$.

ice absorption features, we used a low-order polynomial function to fit and remove the continuum of the ice mixture and the continuum of the spectrum of 2I. Since 2I was observed at high airmass $(>2.0)$, the spectral regions that are heavily affected by the telluric absorption centered at 1.4 and $1.9 \mu \mathrm{m}$ are excluded when fitting the ice features. Studies of Solar System comets have shown that the $1.5 \mu \mathrm{m}$ band in the comet spectra is vulnerable to impurity and particle size effects, and can be absent (Yang et al. 2009, 2014). Therefore, we used the $2 \mu \mathrm{m}$ region to estimate water ice abundance, although the $\mathrm{S} / \mathrm{N}$ is much lower at longer wavelengths, especially in the $K$ band. Using a leastsquares fitting method around $2 \mu \mathrm{m}$, we estimate that water ice abundance in the coma of $2 \mathrm{I}$ is no more than $10 \%$ (Fig. 2).

\subsection{Comparison to other objects}

The spectra of the two known ISOs are shown in Fig. 3a. The spectrum of $2 \mathrm{I}$ is similar to that of 1I. However, we note that there was no coma around 1I, while the nucleus of 2I was buried inside the coma and was not directly detected. Therefore, the spectrum of 2I may not directly reflect the intrinsic composition of the nucleus. At $\lambda<1.4 \mu \mathrm{m}, 2 \mathrm{I}$ shows a red spectral slope that is similar to the mean spectrum of the D-type asteroids. The spectral slope of the 2I spectrum becomes increasingly redder beyond $1.4 \mu \mathrm{m}$, and is redder than those of Jupiter family comets (JFCs) and the Oort Cloud comets (OCCs). This spectral slope increase may be due to the presence of macromolecular organic material (Gradie \& Veverka 1980) that becomes more prominent at longer wavelengths. However, it does not contain the ultra-red
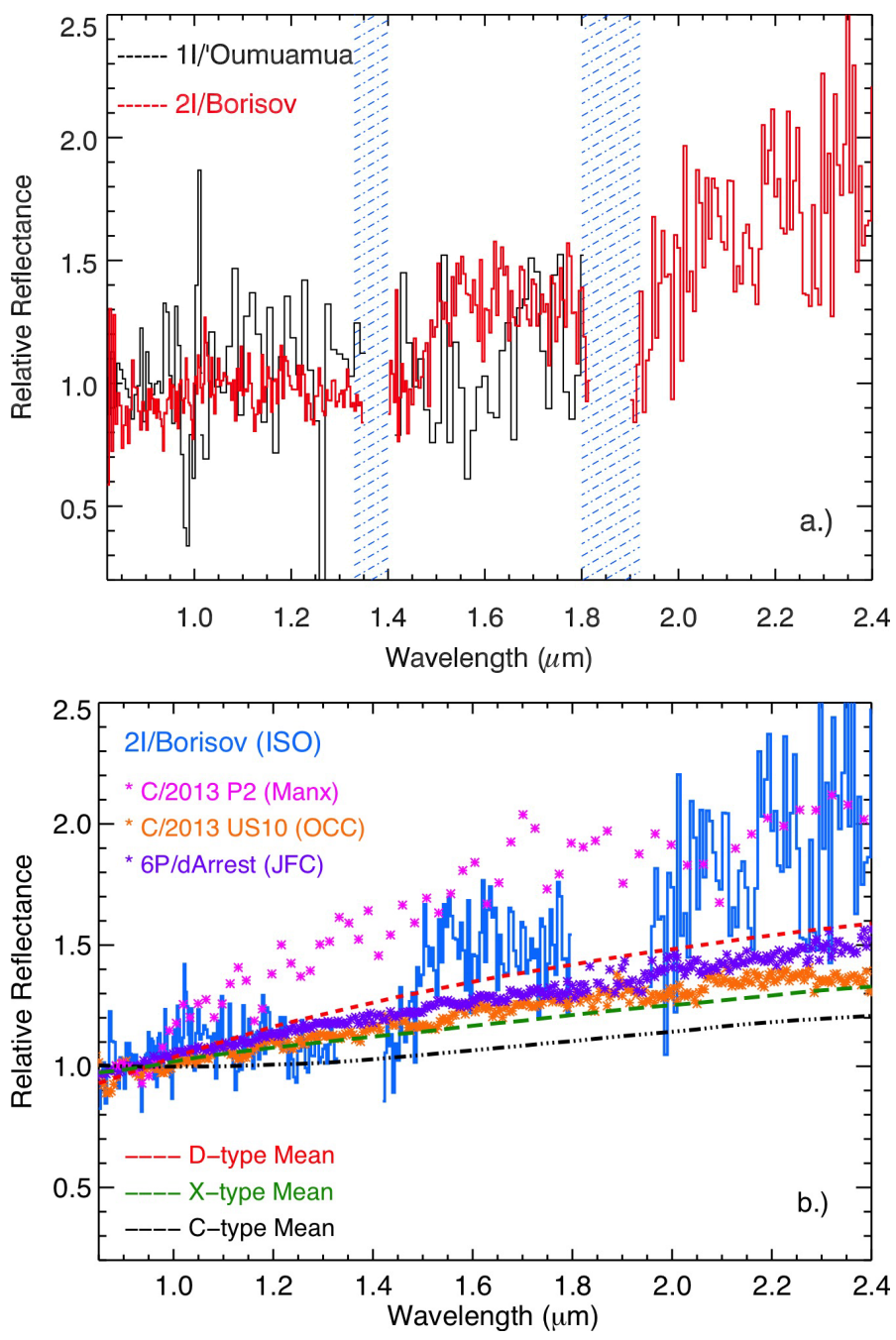

Fig. 3. Panel $a$ : Near-IR spectrum of $2 \mathrm{I} / 2019$ Q4 (Borisov) in comparison with NIR spectrum of 1I/'Oumuamua, taken from Fitzsimmons et al. (2018). Both ISOs appear featureless and the spectral slopes of the two NIR spectra are comparable. Panel $b$ : comparison between the spectrum of 2I and those of Solar System comets and asteroids. The spectrum of C/2013 US10 is from Protopapa et al. (2018), the spectrum of 6P/d'Arrest from Yang et al. (2009), and the three asteroid spectral classes from DeMeo et al. (2009).

material seen in other Solar System bodies (Jewitt \& Luu 2019) and is not as red as the recently detected Manx comet C/2013 P2 (Meech et al. 2014), at shorter wavelengths.

\section{Discussion}

Water ice is a key component of the nuclei of Solar System comets and the primary driver of their activity. However, to date water ice grains in comets (using ground-based telescopes) have only been successfully detected for a limited number of comets, including 17P/Holmes, which showed strong water ice features at 2.0 and $3.0 \mu \mathrm{m}$ after a major outburst (Yang et al. 2009). The survey of 29 individual comets between 1 au and 6 au from the Sun (Protopapa et al. 2015) found it challenging to detect solid water ice features in quiescent comets when the comet is close to or within 2.5 au of the Sun, due to limited grain lifetime. For instance, strong water ice bands were detected when C/2013 US10 (OCC) was beyond 3.9 au from the Sun, and the water ice features disappeared completely even 
in the $3 \mu \mathrm{m}$ region when the comet was $2.3 \mathrm{au}$ from the Sun (Protopapa et al. 2018). The only exception to detecting ice features at a small heliocentric distance is the detection of water ice grains in the innermost coma of comet 103P/Hartely 2 (JFC) by the Deep Impact eXtended Investigation (Protopapa et al. 2014). The well-defined 1.5, 2.0, and $3.0 \mu \mathrm{m}$ water ice absorption features were detected in the spectrum taken $10 \mathrm{~km}$ from the nucleus of 103P when the comet was at 1.06 au from the Sun (Protopapa et al. 2014). There were two populations of water ice grains detected in the coma of 103P: nearly pure water ice with sizes $<5 \mu \mathrm{m}$ and a more distant component of centimetersized icy grains (Kelley et al. 2013). At low ejection speeds, the one-hour lifetime for pure small $(<5 \mu \mathrm{m})$ ice grains ensured that they were present in sufficient numbers to be detected very close to the nucleus (Protopapa et al. 2014). Compared to 103P, 2I/Borisov is much farther away from the Sun and has a much larger effective observing aperture as seen from Earth $(\Delta=3.16 \mathrm{au})$. It is possible that solid ice grains are present in the inner coma, but cannot be detected by the ground-based facilities in a large aperture where the spectrum is dominated by dust.

Following the method described in Protopapa et al. (2018), we estimated the lifetime of water ice grains of various sizes at heliocentric distances of around $2.6 \mathrm{au}$. The balance of energy between absorbed solar radiation, thermal emission, and cooling from sublimation is integrated from a radius of $10 \mu \mathrm{m}$ down to $0.01 \mu \mathrm{m}$, at which point a linear decrease is assumed. For small ice grains with radii of $1 \mu \mathrm{m}$ we found the lifetime of 10000 , 3300 , and $1300 \mathrm{~s}$, corresponding to a refractory carbon impurity of $0.5 \%, 1.0 \%$, and $2.0 \%$ respectively. For large grains with radii of $10 \mu \mathrm{m}$, the estimated lifetime is 65000,9900 , and $4100 \mathrm{~s}$ for the same fractional impurities. The lifetime for grains $100 \mu \mathrm{m}$ in size with $1.0 \%$ carbon is about $2 \mathrm{e} 5 \mathrm{~s}$, and a higher fraction of impurity $(2 \%, 4 \%$, or $10 \%)$ does not affect the lifetime of such large grains significantly. The extraction aperture for the Gemini spectrum is $1.5^{\prime \prime}$ (or $\sim 2830 \mathrm{~km}$ projected in the plane of the sky). Assuming the expansion velocity of the icy grains is represented by the empirical relation: $v_{\text {dust }}=0.535 \cdot r_{h}^{-0.6} \mathrm{~km} \mathrm{~s}^{-1}$ (Whipple 1978), the slit-crossing time is about $9400 \mathrm{~s}$ or $2.6 \mathrm{~h}$. Independently, we modeled the lifetime of icy grains with various albedos and sizes using the method from Meech et al. (1986) and Meech \& Svoren (2004). The derived lifetime of icy grains in comparison to the slit-crossing time is shown in Fig. 4. Since there is no water ice absorption feature observed, we conclude (1) that $10 \mu \mathrm{m}$ radius ice, if present, must have $\gtrsim 1 \%$ carbon fraction; (2) that $1 \mu \mathrm{m}$ radius ice, if present, must have $\gtrsim 0.5 \%$ dirt fraction; and (3) that larger or more pure ice grains are possible, but only if they consist of a small fraction of the coma cross section and are very close to the nucleus.

As discussed previously in Sect. 3.2, unlike the spectrum of 1I, the spectrum of 2I is not from a bare nucleus, but is dominated by the coma surrounding the nucleus. For the case of comet 67P, Snodgrass et al. (2016) noted that the spectral slopes of the coma spectra, obtained via ground-based facilities, are consistent with the values found for the nucleus by the Rosetta Visible InfraRed and Thermal Imaging Spectrometer (VIRTIS). As such, it is not surprising that the two ISOs show comparable spectra and are both lacking the ultra-red matter. Grundy (2009) proposed that mixtures of volatile ice and nonvolatile organics could be extremely red and such ultra-red mixtures become progressively darker and less red as the ice sublimates away. It is possible that 2I originally contained red organic materials that were sublimated or destroyed by the heat and/or radiation of the Sun soon after the ISO entered the inner Solar System.
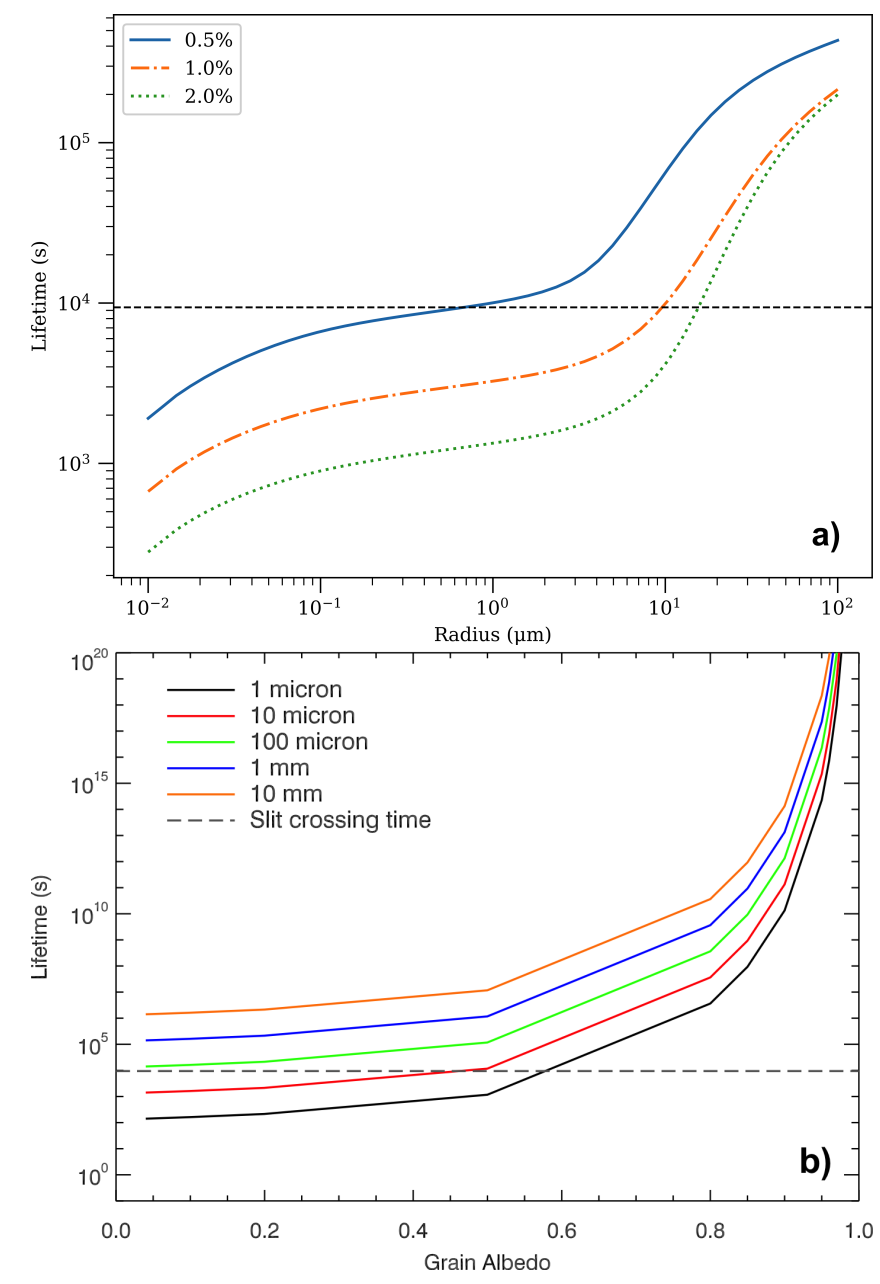

Fig. 4. Lifetimes of water ice grains as a function of grain radius and geometric albedo at the heliocentric distance of $2.6 \mathrm{au}$, as determined from two independent ice sublimation models. The dashed line indicates the slit crossing time of $9400 \mathrm{~s}$.

Unfortunately, no ice was detected and we cannot constrain the original condition of $2 \mathrm{I}$.

Shown in Fig. 1, the spectral slope obtained from the third observation is much flatter than that of the earlier observations. Similarly, the decrease in spectral slope for 1I was detected and discussed in Fitzsimmons et al. (2018), and is attributed to the removal of a surface mantle produced by long-term cosmic ray irradiation. The spectral slope changes as the comet is approaching the Sun may indicate the evolution of organic-rich mixtures in the coma or on the surface of 2I. On the other hand, it is well known that the spectral slope of an active Solar System comet can be affected by the particle sizes in the coma. So the observed slope change in the spectrum of 2I could reflect the change in the particle size distribution in the coma. Bolin et al. (2019) obtained JHK colors of 2I on September 27 and found that 2I exhibits neutral-gray colors in the NIR, which is consistent with our third observation. Since different solar analogs were observed during each observing run, the observed discrepancy in the spectral slope could also be due to the NIR colors of the solar analogs that deviate from the NIR colors of the Sun.

\section{Conclusions}

Our NIR spectrum of 2I/Borisov, taken at the heliocentric distance of $\sim 2.6 \mathrm{au}$, showed no detection of water ice. Based 
on our observations and analysis, we draw the following conclusions:

(1) Large or pure ice grains, if present, comprise no more than $10 \%$ of the coma cross section and most likely reside in the innermost coma of $2 \mathrm{I}$.

(2) $2 \mathrm{I} /$ Borisov displays a moderately red spectrum with a spectral slope of $6 \% / 1000 \AA$ in the NIR, which is similar to D-type asteroids.

(3) No ultra-red matter was observed, which may imply either that 2I did not contain such material originally or that the red material was lost due to the interaction with the Sun.

Acknowledgements. KJM and JVK acknowledge support through awards from NASA 80NSSC18K0853. BY, MSK and SP are visiting astronomers at the Infrared Telescope Facility, which is operated by the University of Hawaii under contract NNH14CK55B with the National Aeronautics and Space Administration. Based in part on observations obtained at the Gemini Observatory [GN2019B-Q-DD-102], which is operated by the Association of Universities for Research in Astronomy, Inc., under a cooperative agreement with the NSF on behalf of the Gemini partnership: the National Science Foundation (United States), National Research Council (Canada), CONICYT (Chile), Ministerio de Ciencia, Tecnología e Innovación Productiva (Argentina), Ministério da Ciência, Tecnologia e Inovação (Brazil), and Korea Astronomy and Space Science Institute (Republic of Korea)

\section{References}

A'Hearn, M. F. 2011, ARA\&A, 49, 281

Bolin, B. T., Lisse, C. M., Kasliwal, M. M., et al. 2019, ApJ, submitted [arXiv: 1910.14004$]$

Cushing, M. C., Vacca, W. D., \& Rayner, J. T. 2004, PASP, 116, 362

DeMeo, F. E., Binzel, R. P., Slivan, S. M., \& Bus, S. J. 2009, Icarus, 202, 160

Elias, J. H., Joyce, R. R., Liang, M., et al. 2006, SPIE, 6269, 62694C

Engelhardt, T., Jedicke, R., Vereš, P., et al. 2017, AJ, 153, 133
Fitzsimmons, A., Snodgrass, C., Rozitis, B., et al. 2018, Nat. Astron., 2, 133 Fitzsimmons, A., Hainaut, O., Meech, K. J., et al. 2019, ApJ, 885, L9

Gradie, J., \& Veverka, J. 1980, Nature, 283, 840

Grundy, W. M. 2009, Icarus, 199, 560

Grundy, W. M., \& Schmitt, B. 1998, J. Geophys. Res., 103, 25809

Hapke, B. 2012, Icarus, 221, 1079

Jewitt, D., \& Luu, J. 2019, ApJ, 886, L29

Kelley, M. S., Lindler, D. J., Bodewits, D., et al. 2013, Icarus, 222, 634

Mastrapa, R. M., Bernstein, M. P., Sandford, S. A., et al. 2008, Icarus, 197, 307

Meech, K. J., \& Svoren, J. 2004, Using Cometary Activity to Trace the Physical and Chemical Evolution of Cometary Nuclei (Univ. of Arizona Press), 317

Meech, K. J., Jewitt, D., \& Ricker, G. R. 1986, Icarus, 66, 561

Meech, K. J., Yang, B., Keane, J., et al. 2014, AAS/Division for Planetary Sciences Meeting Abstracts, \#46, 200.02

Meech, K. J., Yang, B., Kleyna, J., et al. 2016, Sci. Adv., 2, e1600038

Meech, K. J., Weryk, R., Micheli, M., et al. 2017, Nature, 552, 378

Micheli, M., Farnocchia, D., Meech, K. J., et al. 2018, Nature, 559, 223

Min, M., Bouwman, J., Dominik, C., et al. 2016, A\&A, 593, A11

Mumma, M. J., \& Charnley, S. B. 2011, ARA\&A, 49, 471

Opitom, C., Fitzsimmons, A., Jehin, E., et al. 2019, A\&A, 631, L8

Protopapa, S., Sunshine, J. M., Feaga, L. M., et al. 2014, Icarus, 238, 191

Protopapa, S., Kelley, M. S. P., Yang, B., et al. 2015, AAS/Division for Planetary Sciences Meeting Abstracts, \#47, 415.08

Protopapa, S., Kelley, M. S. P., Yang, B., et al. 2018, ApJ, 862, L16

Raymond, S. N., Izidoro, A., \& Morbidelli, A. 2018, ArXiv e-prints [arXiv:1812.01033]

Rayner, J. T., Toomey, D. W., Onaka, P. M., et al. 2003, PASP, 115, 362

Rouleau, F., \& Martin, P. G. 1991, JRASC, 85, 201

Shannon, A. B., Jackson, A. P., Veras, D., \& Wyatt, M. 2015, AAS/Division for Extreme Solar Systems Abstracts, \#47, 109.07

Snodgrass, C., Jehin, E., Manfroid, J., et al. 2016, A\&A, 588, A80

Walsh, K. J., Morbidelli, A., Raymond, S. N., O’Brien, D. P., \& Mandell, A. M. 2011, Nature, 475, 206

Whipple, F. L. 1978, Nature, 273, 134

Yang, B., Jewitt, D., \& Bus, S. J. 2009, AJ, 137, 4538

Yang, B., Keane, J., Meech, K., Owen, T., \& Wainscoat, R. 2014, ApJ, 784, L23

Ye, Q.-Z., Zhang, Q., Kelley, M. S. P., \& Brown, P. G. 2017, ApJ, 851, L5

Ye, Q., Kelley, M. S. P., Bolin, B. T., et al. 2019, AJ, accepted [arXiv:1911.05902] 